\title{
Readability and Health Communication: An Analysis of the Readability of Commonly Used Malaria Drugs Information Leaflets in Cape Coast, Ghana.
}

\author{
William Kodom Gyasi \\ Department of Communication Studies, University of Cape Coast, Ghana
}

\begin{abstract}
This study conducted in-depth analysis of the readability of commonly used malaria medicine information leaflets in Cape Coast, Ghana. Seven leaflets of malaria medicines that are very popular in Cape Coast, Ghana were analyzed using the Flesch-Kincaid Reading Ease and Gunning Fog readability indexes. These leaflets were also examined based on the legibility of print, type of paper and bilingual information. Data collected from the two indexes revealed that all the leaflets are very difficult to read. Values ranged from 10.4 to 38 for the Flesch-Kincaid Reading Ease indicating that the leaflets are very difficult to comprehend and that one needs university education to comprehend them.14.2 to 18.8 were recorded for the Gunning Fog index also indicating that the leaflets are very difficult and that in some cases are even not readable for people with university education. The mean value for the readability consensus for the two readability indexes was 21.04. The implication is that in terms of the Gunning Fog index, the leaflets are very difficult to comprehend and that they were written far above the reading comprehension level for university graduates. Also, in the case of the flesch reading ease regarding the readability consensus of the two indexes, the leaflets are very difficult to comprehend in terms of level of reading difficulty but match the appropriate reading level of the university graduate. The findings of the study also revealed that 5 leaflets (71.4\%) had a font type size $<10$ and none (0\%) was produced in any of the major languages in Ghana apart from English and French. Conclusively, the study discovered that malaria medicine information leaflets available in drug stores and hospitals in Cape Coast, Ghana are not readable to the majority of the population. The significance of rendering the valuable information contained in those leaflets comprehensible to consumers is life and death matter. Hence, pharmaceutical companies in Ghana are encouraged by the researcher to produce readable medicine information leaflets so that their intended purpose can be achieved.
\end{abstract}

Keywords: Malaria, Medicine information leaflets, Readability, Flesch-Kincaid Reading Ease, Gunning Fog.

\section{Introduction}

In spite of the considerable efforts throughout the century to eradicate or control malaria, it is still the most prevalent and most devastating disease in the tropics. According to the 2011 Ghana Health Service Report (GHS), malaria remains hyper endemic in Ghana, accounting for about 32.5\% of all outpatient illnesses, 35.9\% of all admissions, and 30.3\% of all deaths in children under age five (cited in Akapule, 2011). In other words, it means more than 17 million out of 24 million Ghanaian are infected by malaria every year, costing the nation 85,000 Ghana cedis (US\$94 million) for treatment alone (Akapule,2011). Malaria is estimated to cause the loss of about $10.6 \%$ Disability Adjusted Life Years (DALYs) costing an equivalent of up to 6\% of GDP annually in economic burden (Akapule, 2011). Therefore, the Ghana Poverty Reduction Strategy II (GPRS II, 2003-2009) identifies malaria control as one of the key health sector interventions.

Buabeng et al. (2007) intimated that in Ghana, most malaria cases are managed at the household level. Help is usually sought from the community pharmacist or licensed chemical seller (registered suppliers of specified over-the-counter-medicines) after the initial therapy has failed. Most patients reporting at clinics and hospitals facilities would have gone through home-based treatment or community drug shops/pharmacies initially. Thus, mainly resistant, severe, or recurrent episodes are seen at clinics and hospitals. There is concern that community and household handling of malaria is often inadequate, inappropriate and ineffective (Buabeng et al., 2007).

By means of several strategies, the Ghana Health Service has created awareness about the deadly nature of malaria. One of such strategies is the provision of prompt and effective case management of malaria cases. In collaboration with World Health Organization, USAID, Roll Back Malaria Initiative and The Global Fund, Ghana Health Service has provided an up-to-date manual for health care workers nationwide in connection with malaria treatment. A key strategic components included in this manual are adequate and practical information on how to heighten delivery of antimalarial medicines at home and community levels (Ghana Health Service, 2009). Also, the manual provided extended explication on Health promotion and 
behaviour change communication; and appropriate supply of good and quality Artesunate and Amodiaquine or Artemether Lumefantrine (AL) and Dihydroartemesin in Piperaquine (DHAP).

In most severely hit countries in Africa, including Ghana, according to Buabeng et al., (2007) increasing reports of widespread resistance to chloroquine and other common antimalarial drugs have necessitated a change in policy to Artemisinin Combination Therapy (ACT) as first line treatment for uncomplicated malaria. In Ghana, the recommended ACT is artesunate-amodiaquine. This has huge economic implications for most poor patients in sub-Saharan Africa, since the ACTs are comparatively more expensive ( Akosah 2007, Akapule 2011). However, a national health insurance scheme has been instituted in Ghana supported by an act of parliament that allows the public to access health care for diseases of public health concern without making an out-of-pocket payment for attending a health institution or paying for drug prescriptions. Effective implementation of this scheme may provide relief economically for use of ACT for uncomplicated malaria in Ghana.

With the introduction of ACT's as over-the-counter (OTC) medicine to promote home management, it is important to ensure appropriate usage of these medicines to prevent development of resistance to the artemisinins and related combination products, and to reduce cost associated with treatment failures and also health complications. Hence, according to Bernard and Sparks (1993) appropriate medicine information is important to ensure their rational use at the community level. Medicine information leaflets should be written with appropriate font size, language and readability. For example, this article was written with Times New Roman font size 12 which I believe is readable.

In order to provide a simple and clear definition of readability as suggested by Readability formulas.com (www.readabilityformulas.com), one can dissect the term "readability" into "read" + "ability," which translates into the ability to read. In fact, readability is more of a judgmental exercise for the targeted audience. Klare (1984) defines readability as "the ease of understanding or comprehension due to the style of writing." Readability can be considered as means to measure the difficulty of text or page layout, so the writer knows how effectively his text will reach his target audience before he publishes or distributes it.

Assessing readability requires the use of conducting readability tests through the use of readability formulas or indexes. Readability indexes are mathematical in nature; each formula's primary aim is to measure the grade level or years of schooling a person must have to read and comprehend a text. Writers consider readability formulas as simple ways to judge readability, i.e., the level of difficulty of a text. Readabilityformulas.com indicates that readability formulas work by measuring certain features of a text based on mathematical calculations. According to Macdonald, McMillan, and Kerr (2010), these formulas often use word length, sentence length, and polysyllabic frequency. Flesch-Kincaid Reading Ease Readability Index and the Gunning Fog index are two commonly used readability formulas.

These two formulas are commonly used in estimating the level of reading comprehension difficulty of the content of document including medicine information leaflet. Authenticating its reputation for reading level accuracy, Ivan (2010) posits: "Gunning's Fog Index is one of the best known readability indexes and measures the level of reading difficulty of any document." Regarding Flesch-Kincaid Reading Ease, readabilityformulas.com stated: Flesch Reading Ease Formula is considered as one of the oldest and most accurate readability formulas.

\section{Importance of Readability in Health Communication}

The importance of Healthcare Industry cannot be overemphasized because a vibrant healthcare industry ensures a healthy nation, vibrant economy and a flourishing lifestyle for its citizens. It is vital to adopt simple modes of communications between healthcare professionals and patients. One such way to improve communications in healthcare is by using readability formulas on documents as a means to communicate effectively with patients and health care suppliers.

Since uncontrolled, immoderate and inappropriate in-take of medicine can be poisonous to the human body, medicine leaflet without appropriate readability in simple terms and comprehensibility can cause a lot of havoc. For example, it was stated in one of the malaria medicines:

Do not take Artesunate Amodiaquine Winthrop - If you have ever had liver problems during treatment with amodiaquine. - If you have ever suffered from sudden high fevers, shaking, severe sore throat or ulcers in the mouth (symptoms suggestive of harm to white blood cells) during treatment with amodiaquine. -If you have an eye disease with damage to your retina. - If you have any doubt, it is essential to ask the advice of your doctor, pharmacist or health care worker. Take special care with Artesunate Amodiaquine Winthrop. This medicine may cause damage to the liver and/or blood that can sometimes be fatal. 
Just imagine how dangerous it will be for an individual who has any of these health conditions takes any of these drugs if he does not understand the content of the leaflets. Hence, it is not only right to subject medicine leaflets to readability index analysis to ensure they are comprehensible to the average reader but it is also a moral obligation. With the help of readability indexes, there can be improvement in patient education and awareness. Patients need to understand their ailments, prescribed drugs, risks and side effects of the drugs, clinical tests, when to contact their doctors, when to seek immediate medical attention, and so on. All these can be accomplished by communicating with the patient through written communications, verbal instructions, multimedia, etc. Many patients need written instructions for long-term reference, and they need to understand their healthcare material in simple terms. Using readability formulas can help hospitals prepare documentation that patients can read and understand at their reading-level.

For overall effective management of disease, it is vital the doctor-patient-pharmacist communication is as clear as possible. If the written text is beyond the reading abilities of the patient, he cannot understand the measures he needs to take to manage his disease. If the pharmacist can't understand the prescription from a doctor, there's every chance the pharmacist could give a wrong medication to the patient. Readability formulas can help health professionals prevent such unfortunate events.

\section{Statement of the Problem}

In the past, several studies were conducted in many countries to set proper guidelines and regulations on readability and design of medicine information leaflets. The results of such findings revealed that several medicine information leaflets are above the reading level of the target population. Steps were taken to correct such rendition and presently, there are many countries with appropriate medical information leaflets for its citizenry. Such studies included the in-depth analysis of the readability of medicine information leaflet in the United States by Basara and Juergens (1994). Their findings revealed that most of the medicine information leaflets in the United State are above the suggested readability level of fifth-to-seventh grade. An insightful study was also conducted by Bradley et al. (1994). Their study assessed the readability of over-the-counter medicine information leaflets available in the United Kingdom market and concluded that the mean reading grades obtained were above the mean reading age of the general adult population. In South Africa, Mansoor and Dowse (2003), also did a thorough examination of the medicine information leaflets and their report was instrumental to improving the readability of medicine information in the country. Again, in their study, Chubaty et al. (2009) demonstrated that most medicine leaflets intended for seniors in Canada, did not meet established guidelines.

More importantly, an in-depth study has been conducted in Nigeria by Auta et al. (2011) in relation to readability of malaria medicine information. During the course of the study, forty-five malaria medicine information leaflets were subjected to a thorough readability test using the SMOG readability index. They had a resounding success in their study and relevant recommendations were made. However, the researcher is unaware of any such study conducted to assess or analyze the readability of medicine information leaflets in Ghana. Hence, it would be pleasing to conduct in-depth analysis of popular malaria medicine information leaflets in Ghana.

\section{Limitations of readability formulas}

The present study conducted in-depth analysis of medicine information readability using two readability indexes - Flesch-Kincaid and Gunning Fog. It should be noted that certain limitations are associated with readability indexes. According to Bernard et al. (1993), Smith (1998), and Readabilityformulas.com (2013), readability formulas do not consider readers' motivation and familiarity with certain vocabulary and can sometimes overestimate the difficulty of the passage. However, according to Stephens (2000), readability formulas can serve as an early warning system to let the writer know that the writing is too dense. They can give a quick on-the-spot assessment. They have been described as "screening devices" to eliminate dense drafts and give rise to revisions or substitutions (Klare, 1976, p. 8, 129-152). Also, in some organizational settings, readability tests are considered useful to show measurable improvement in written documents. They provide a quantifiable measure of improvement or simplification (Stephens, 2000)

\section{Test samples}

\section{Methodology}

Malaria medicine information leaflets were obtained from five doctors. The five doctors were drawn from the University of Cape Coast Hospital and central regional hospital. Both hospitals are in the central region of Ghana. Only malaria medicine information leaflets in respect of antimalarials of Artemisinin Combination Therapy (ACT) of which artesunate-amodiaquine was used and those registered by Ghana Food and Drugs Board (FDB) were included in the study. Each doctor provided a list of malaria medicines they often prescribed for their patients. It was observed that seven medicines were common to the lists of the five doctors. These 
seven medicines were taken to two well patronised pharmacy shops in the cape coast metropolis of Ghana and the shop attendants confirmed the fact that these drugs are often patronized with prescriptions from doctors and indeed, they also frequently sell them to patients without prescription.

\section{Readability Test using Two Readability Indexes}

The Flesch-Kincaid Reading Ease and Gunning Fog Scale were used to ascertain the reading ease and level of comprehensibility of the selected medicine information leaflets. Various sections of the information leaflets were fed to the online (automated) readability indexes provided by Readabilityformulas.com, so as to generate reading ease and level of difficulty of understanding or comprehending each of the leaflets.

According to Braught (2003), the Flesch Reading Ease Index will output a number from 0 to 100. Readability score of $90-100$ can be read and understood by $5^{\text {th }}$ graders (pupil in class five) while 8th and 9th graders can understand documents with a score of 60-70. University graduates can understand documents with a score of 0-30 (Braught ,2003). In terms of the level of reading difficulty, documents that score a Flesch Reading Ease of 0-30 are considered very difficult while those that score 30-50 are considered difficult. A Flesch Kincaid reading ease of 50- 60 is for documents that are fairly difficult and 60-70 score is for standard documents. A Flesch reading ease score of 70-80 and 90-100 are ascribed to easy and very easy documents respectively Grundner, (1978), Rush (1985),Meade and Smith (1991).

Writers are encouraged to aim for a score of approximately 60 to 70. Gunning Fog compares syllables and sentence lengths. The scores are usually a rough measure of how many years of schooling it would take someone to understand the content of a reading material or the level of reading difficulty of a text. A Fog index of 1-8 is for elementary school students, while 9-12 is for high school students. A document that scores a fog index of 13-16 is for post secondary school students. The lower the number, the more understandable the content will be. The ideal score for readability with the Fog index is 7 or 8 . Anything above 12 is too hard for most people to read ((Grundner, T.M. (1978), Rush, R.T (1985), Meade and Smith (1991)

\section{Other Considerations}

Since font and language use are also a contributory factor to readability, the researcher also examined the leaflets for font type and size, and bilingual information. Font size was evaluated by comparing the font size of each leaflet with a printed document containing different theme fonts of size 10. Font size of 10 was considered by the researcher as the minimum acceptable font size that is easy to read. Interestingly, some journals indicate in their submission requirement that articles submitted for publication should have a font size of 10 .

\section{Statistical analysis}

The researcher analyzed the emerging data using Statistical Package for Social Sciences (SPSS) version 16 to generate the mean and standard deviation of the information leaflets' reading ease as well as the frequencies and percentages for all variables. Then a mean value was obtained based on the values of the two indexes.

\section{Results And Discussion}

Table 1: Malaria medicine information leaflets' reading levels using the Flesch-Kincaid Reading Ease and Gunning Fog readability indexes

\begin{tabular}{lll}
\hline $\begin{array}{l}\text { Flesch-Kincaid } \\
\text { Reading Ease }\end{array}$ & Gunning Fog & Readability Consensus \\
\hline 24.8 & 17.3 & Very difficult to read \\
36.3 & 14.2 & Difficult to read \\
21.1 & 14.5 & Very difficult to read \\
16.2 & 18.6 & Very difficult to read \\
10.4 & 18.8 & Very difficult to read \\
36.3 & 15.3 & Difficult to read \\
36 & 14.5 & Difficult to read \\
\hline
\end{tabular}

$\mathrm{n}=7$

Source: Field work, 2013.

As shown in Table 1, all the medicine information leaflets under consideration are very difficult to read with values ranging from 10.4 to 36.3 for the Flesch Kincaid Reading Ease and 14.2 to 18.8 for the Gunning Fog index. Thus, in the case of the score for the Flesch reading ease, a reader must have a university education in other to comprehend the content of the leaflets. As shown by the table, the gunning Fog score even exceeds the grade level requirement for university graduate readers which is 13 to 16 (Grundner, 1978), (Rush, 1985), (Meade and Smith 1991) 
The readability consensus value (result of readability test based on the average of two readability indexes. Readabilityformulas.com has an automated readability consensus value for several indexes) was 21.04 with a standard deviation of 4.55. Clearly when considered from the Flesch Reading Ease perspective, the leaflet can be understood when one has a university education. When measured in terms of the level of reading difficulty, the leaflets are very difficult (Grundner, 1978), (Rush, 1985), (Meade and Smith 1991).

In the case of the gunning fog index, however, the leaflets are far above the appropriate reading level for post university readers which are 13-16, hence very difficult to comprehend.

\section{Legibility of Print Test}

The researcher tested the leaflets for legibility and it was revealed that 2 representing $28.6 \%$ were written with font size 10 while the majority, 5 representing $71.4 \%$ were written with font size lesser than 10 $(<10)$. The implication is that when readability of the leaflet are considered from the point of legibility of print, they are generally not readable especially for those with sight problems. Furthermore, the researcher checked the type of paper used. It was shown that all the 7 leaflets representing $100 \%$ are non-glossy and uncoated papers. This is very good because it enhances readability.

\section{Other Test}

The researcher again analyzed the leaflets on the bases of type of language used and pictorial illustrations. The study revealed that none of the leaflets has pictorial illustration.

The languages used are shown in Table 2 below:

Table 2: Bilingual Data

\begin{tabular}{|c|c|}
\hline Language & Percent \\
\hline English language only & $1(14.28 \%)$ \\
\hline English language + International language & $6(85.72 \%)$ \\
\hline English + Local language & None $(0 \%)$ \\
\hline
\end{tabular}

Source: Field Survey. 2013.

\section{Findings}

As depicted in Table 1, the readability consensus revealed that 5 representing $57.1 \%$ of the leaflets are very difficult to read, while $42.9 \%$ are considered difficult to read. The result shows that the information contained in most of the medicine information leaflets are basically very difficult to understand by an average Ghanaian. Essentially, malaria information leaflets are to inform and create awareness about pertinent matters regarding the use of the medicine. Therefore, they should be made highly readable for easy comprehension for the average Ghanaian. This is important because in Ghana these medicines are often obtained over the counter without doctor's prescription and many people are not proficient in the English language (Owu-Ewie, 2006). Having readable malaria medicine information leaflets will improve rational use of the drugs in home-based handling of malaria. In connection with health information, Nightingale (1995) affirms that readable and comprehensible consumer information improves rational use of medicines.

The high scores obtained in this study for both Flesch-Kincaid Reading Ease (10.4), and Gunning Fog (18.8), is as a result of sentence length and use of complex grammatical structures and medical/pharmaceutical terminology which lead to incomprehensible medicine information leaflets. Such complex language in information leaflets, according to Raynor et al. (2005) has been reported to be of concern to consumers because majority are not familiar with medical/pharmaceutical terminology, in fact, they often confuse consumers.

In their research, Raynor et. al. (2005) also indicated that consumers find it difficult to read leaflets with small font; this may be due to the fact that some people suffer varied degree of sight problems, such as long or short sightedness. As indicated under legibility of print, findings in this study revealed that $71.4 \%$ of the leaflets were written with very small font size of less than $10(<10)$ which undoubtedly makes them illegible or difficult to read for people with impaired sight. Hence, when designing medicine information leaflets, it is essentially important to consider legibility of the printed material because it also contributes to readability of the leaflets. Regarding the type of paper used, the study revealed that $100 \%$ of the leaflets used papers that are not coated or glossy; this is good, because such papers do not reflect light, making it easier to read (Dietrich, 2013).

The study further revealed that all the malaria medicine information leaflets examined do not have any pictorial illustration. Commenting on the importance of pictorial illustration, Hwang (2005) posits: "pictorial illustrations can improve comprehension of medicine information if the illustrations and text are well-matched to each other and are appropriate to the educational and cultural background of the user". Hence, writers of medicine information leaflets should generously use pictorial illustrations to buttress and underscore vital information contained in preparing medicine information leaflets. 
Furthermore, the researcher also checked the language used and as depicted in Table 2, 85.72\% of the leaflets were written in English and an international language. This means that local languages were sidelined. Probably, due to the country's linguistic ecology. Although, English has come to stay as the lingua franca in Ghana, however, in tune with Buck (1998), writing medicine information in consumers' first language or mother-tongue is a time-proven strategy facilitating readability. People naturally think or process information in their primary language, hence they easily understand and comprehend matters written in their primary language. English and other international languages can be supplemented with some local languages to aid and improve readability.

\section{Conclusion}

The analytical approach adopted in the study yielded some findings in connection with malaria information leaflets. The findings revealed that malaria medicine information leaflets available in the Ghanaian market are very difficult to comprehend by the average Ghanaian (Table 1). The readability consensus of the two readability indexes - Flesh Kincaid Reading Ease and Gunning Fog index was 21.04, which means the leaflets were written beyond the appropriate readability levels for even university graduates in some cases. Also, the study revealed that there is need for pharmaceutical companies registered under the Ghana Food and Drugs Board to design readable and comprehensible malaria medicine leaflets. This matter should be enforced by the Food and Drugs Board in Ghana. This has become necessary because ACTs (Artesunate-Amodiaquine) is an over-the counter drug (OTC) and can be accessed by anyone without the prescription of a doctor. Moreover, the proficiency level of a large section of the population is very low.

\section{Recommendation}

In view of the findings and conclusions of the study, the following recommendations are appropriate:

a. The study revealed that the information contained in the malaria medicine leaflets were written beyond the comprehension level of an average Ghanaian. In view of this, pharmaceutical companies in Ghana should produce malaria medicine information leaflets at the reading level of an average Ghanaian through the use of plain language.

b. Ghana Health Service in collaboration with the Food and Drugs Board should also formulate and enforce policies that would ensure that certified pharmaceutical manufacturing companies within and outside Ghana produce malaria medicine information leaflets that are less technical, functional and readable.

\section{References}

[1]. Akapule, S. A., (2011). Malaria Bites into Economic Development, Ghana News Agency.

[2]. Akosah, K. (2007) Fighting Malaria in Ghana. International Society for Individual Liberty, http://www.isil.org/resources/fnn/2007june/malaria-ghana.html. Retrieved April 9, 2013.

[3]. Auta, A., Shalkur, D., Banwat, S. B. \& Dayom, D. W. (2011) Tropical Journal of Pharmaceutical Research, 10 (5): 631-635.

[4]. Bernard, I., Sparks I. I. (1993) Readability of ophthalmic literature. Optometric Vision Science, 70(2): 127-130.

[5]. Bradley, B., Singleton, M., Po, ALW. (1994). Readability of patient information leaflets on over- the-counter (OTC) medicines. J Clin Pharm Ther., 19: 7-15.

[6]. Buabeng, K. O., Duwiejua, M., Dodoo, A. N., Matowe, L. K. \&Enlund, H (2007). Self - reported use of anti-malaria drug and health facility management of malaria in Ghana. Malaria Journal 6:85 doi: 10.1186/1475-2875-6-85

[7]. Buck, M. L. (1998) Providing patients with written medication information. Ann Pharmacother., 1998; 32:962-969.

[8]. Chubaty, A., Sadowski, C. A., Carrie, A. G. (2009) Typeface legibility of patient information leaflets intended for communitydwelling seniors. Age Ageing, 38(4): 441-447.

[9]. Deiterich, A. (2013) Types of Glossy Papers. eHow.com. http://www.ehow.com/ about_5368935_types-glossy-paper.htm

[10]. Ghana Health Services Report (2009). Manual for health care workers nationwide in connection with malaria treatment www.ghanahealthservice.org/...TRAINING\%20MANUAL\%20FOR\%20 Hf.pdf. Retrieved March 13, 2013.

[11]. Hwang, S. W., Carolyn, Tram, Q. N. \& Knarr, N. (2005) The effect of illustrations on patient comprehension of medication instruction labels. BMC Family Practice, doi:10.1186/1471-2296-6-26

[12]. Klare, G. (1984) (Eds.). Readability. In P.D. Pearson, R. Barr, M.L. Kamil, and P. Mosenthal Handbook of reading research (pp. 681-744). New York: Longman.

[13]. Ivan, A. (2010). Klariti.com. Fog Index and Readability Formulas. Available at http://www.klariti.com/business-writing/FogIndex-Readability Formulas.shtm1\#top.. Accessed on March 12, 2013.

[14]. Macdonald, S., McMillan, T. M., Kerr, J. (2010) Readability of information leaflets given to attenders at hospital with a head injury. Emergency Medical Journal, 27:279-282

[15]. Mansoor, L. E., Dowse, R. (2003) Effect of pictograms on readability of patient information material. Ann Pharmacother., 37(7): 1003-1009.

[16]. Meade, C.D., \& Smith, C.F. (1991). Readability formulas: Cautions and criteria Nightingale S. L. (1995) Written patient information on prescription drugs. Int J Technol Assessment Health Care, 11(3): 399-409.6.

[17]. Owu-Ewie, C. (2006). Selected Proceedings of the 35th Annual Conference on African Linguistics, ed. John Mugane et al., 76-85. Somerville, MA: Cascadilla Proceedings Project.

[18]. Raynor, D. K., Blenkinsopp, A., Knapp, P., Grime, J., Nicolson, D. J., Pollock, K., Dorer, G., Gilbody, S., Dickinson, D., Maule, A. J, et al. (2007) A systematic review of quantitative and qualitative research on the role and effectiveness of written information available to patients about individual medicines. Health Technol Assess, 11(5): 1-160.

[19]. Readabilityformulas.com. http://readabilityformulas.com/. Retrieved March 22, 2013.

[20]. Rush, R.T. (1985). Assessing readability: Formulas and alternatives. The Reading Teacher, 39, $274-283$. 


\section{APPENDIX \\ RESULTS OF READABILITY TESTS FOR \\ MALARIA MEDICINE INFORMATION LEAFLETS}

\section{BRAND NAME: ARTEMOS-PLUS}

Artemos-Plus is an antimalarial ACT accordance with WHO's recommendation, the combination of artemether and lumefantrine, which acts as a blood schizontocide. it is indicated for: Treatement, including stand-by emergency treatment of adults, children and infants with acute, uncomplicated infections due to plasmodium falciparum or mixed infections including P. falciparum. Because Artemos-Plus is effective against both drug-sensitive and drug-resistant $\mathrm{P}$. falciparum. it is also recommended for malaria infections acquired in areas where the parasites may be resistant to other antimalarials. Stand-by emergency treatment: Most tourists and business travelers, considered to be non-immune, will be able to obtain prompt medical attention if malaria is suspected. However, a minority at risk of infection maybe unable to obtain such care within 24 hours of the onset services. In such cases, prescribers are advised to issue Artemos-Plus to be carried by the travelers for self-administration. ("stand-by emergency treatment"). Consideration should be given to official guidance regarding the appropriate use of antimalarial agents.

\section{RESULTS:}

Flesch Reading Ease score: 24.8 (text scale)

Flesch Reading Ease scored your text: very difficult to read.

Gunning Fog: 17.3 (text scale)

Gunning Fog scored your text: difficult to read.

\section{BRAND NAME: LONART}

With Artemether virtually no side effect have been seen. Laboratory abnormalities such as slight rise in tranaminases and a decrease in reticulocyte count are rare and transient. A lowering of sinus frequency without causing ECG changes has been noticed. At high does transient abdominal pain, tinnitus and diarrhea have been described but a causal relationship is unclear. Some antimalaria as halofantrine and quinine can influence the ECG pattern. Attention should be made to patients previously treated with those antimalarials. A reasonalbe period should be taken in account before to start treatment with lumefantrine combinations. For those patients physicians will be prescribed Artemisinin derivatives in mono therapy in cause of severe paludism. Sometimes it could be possible that the following common side effect occur: rash, check this with you doctor. Other common side effects may occur as trouble of sleeping, nausea, vomiting, diarrhea, coughing. They need medical attention when persisting. Resistance and recrudescence: Resistance of Plasmodia to artemether has not been observed. It is also unlikely to occur in view of the specific mechanism of action which is very cytotoxic for Plasmodia (opening of a peroxide bridge). An apparent resistance is sometimes seen but is mainly due to multiple broods of plasmodia developing at different times in the same patient. In controlled studies recrudescence does not exceed $10 \%$. In case of recrudescence (renal or apparent) a new complete treatment for three days is advisable.

\section{RESULTS:}

Flesch Reading Ease score: 36.3 (text scale)

Flesch Reading Ease scored your text: difficult to read.

Gunning Fog: 14.2 (text scale)

Gunning Fog scored your text: hard to read.

\section{BRAND NAME: P-ALAXIN}

Mode of action of Dihydroartemisin: Dihydroartemisin mainly interferes with the membrane structures of trophozoites (erythrocytic asexual forms), i.e whorled food vacuole membrane, distended mitochondria, swollen nuclear membranes, dissociation of ribosomes from endoplasmic reticulum leading to cytoplamic vacuolization and autophogocytosis. In addition, biochemical depression of protein synthesis and nucleic acid synthesis are exhibited. Upon oral administration Dihydroartemisin is rapidely absorbed and maximum blood concentration attained 1 hour afterwards, with a half-life of about 4 hours. It is widely distributed in the liver, kidneys and bile. Approximately $80 \%$ is excreted through the urine and feces within $24 \mathrm{hrs}$ after administration. it is metabolized to two inactive metabolites, deoxydihydroartemisinin and dihydroxydihydroartemisinin. Experimental results show that PQP interferes with physiological function of the food vacuole membrance of the trophozoites leading to autophagocytosis of the parasites. It has no marked effect on the ring forms, immature or mature schizonts and the male or female gametocytes. Upon oral administration about $80-90 \%$ is absorbed within $24 \mathrm{hrs}$. It is widely distributed in the body mainly in the liver, kidneys, lungs and spleen. 
About $25 \%$ of the total dose is partitioned in the liver within $8 \mathrm{hrs}$ of intake. Elimination is very slow with the half life of about 9.4 days. It is excreted through the bile by hepatoenteral circulation.

\section{RESULTS:}

Flesch Reading Ease score: 21.1 (text scale)

Flesch Reading Ease scored your text: very difficult to read.

Gunning Fog: 14.5 (text scale)

Gunning Fog scored your text: hard to read.

\section{BRAND NAME: ARSUAMOON}

Pharmacology and toxicology: The product is a combination preparation of Artesunate Tablets and Amodiaquine Tablets. Artesunate targets the structure of plasmodium, the main mode of action being interference with the function of pellicle-mitochondria. It may be the cause of Artesunate effecting on the food vacuole that leads to the blocking of the nutritive intake at an early stage, leading to a fast occurrence of amino acid deficiency and quick formation of autophagic vacuole and eject of plasmodium. So, the plasmodium dies from losing a large part of the cytoplasm. Amodiaquine accumulates in the parasite's food vacuole and inhibits heme polymerization, thereby increasing its efflux out of the food vacuole. The drug competitively inhibits the degredation of heme by glutathione, thus allowing heme accumulation in the membrane and permeates it to cations, thereby disturbing cation homeostasis in the parasite, leading to parasite death. The combination tablets have good synergistic action, which is also active against chloroquine resistant plasmodium. The results of toxicological experiments in animkals showed that Artesunate had embryo-toxicity. The toxicity rate of Amodiaquine is LD50 (mouse) $225 \mathrm{mg} / \mathrm{kg}$ intraperitoneally, and LD50 (mouse) $550 \mathrm{mg} / \mathrm{kg}$ orally.

\section{RESULTS:}

Flesch Reading Ease score: 16.2 (text scale)

Flesch Reading Ease scored your text: very difficult to read.

Gunning Fog: 18.6 (text scale)

Gunning Fog scored your text: difficult to read.

\section{BRAND NAME: ARTEFAN}

Pharmacodynamics: Artefan comprises of Artemether and Lumefantrine in a fixed ratio of 1:6 respectively. The food vacuole of the malarial parasite is the site of antiparasitic action for both Artemether and Lumefantrine, where they are thought to interfere with the conversion of haem, a toxic intermediate produced produced during hemoglobin breakdown, to the nontoxic haemozoin, malaria pigment. Lumefantrine is thought to interfere with the polymerization process, while Artemether generates reactive metabolites as a result of the interation between its peroxide bridge and haem iron. They also have a secondary action which involves the inhibition of the nucleic acid and protein synthesis within the malarial parasite. In-vitro and in-vivo studies show that Artemether and Lumefantrine combination does not induce resistance. The combination of Artemether and Lumefanrine into Artefan has enhanced the independent antimalarial activity of both, which has been shown to potentiate the blood schizonticidal effects. It is also effective against drug resistant strains of $P$. falciparum malaria. These two components have shown marked synergy during comprehensive invitro studies using laboratory maintained and fresh field parasite isolates from different malaria endemic areas. The combination also clears gametocytes more rapidly than non-artemisinin antimalarials as per results of comparative clinical trials.

\section{RESULTS:}

Flesch Reading Ease score: 10.4 (text scale)

Flesch Reading Ease scored your text: very difficult to read.

Gunning Fog: 18.8 (text scale)

Gunning Fog scored your text: difficult to read.

\section{BRAND NAME: LUMARTEM}

Possible Side Effects: Like all medicines, Lumartem can cause side effects, although not everybody gets them. When treating malaria. it is not always possible to differentiate between unwanted effects caused by Lumartem, and those caused by any other medicines you may be taking at the same time. For this reason, it is important that you inform the doctor or health care provider of any change in your health. The following side effects have been reported in patients treated with Lumartem. The most commonly reported side-effects (greater than 1 in every 10 patients treated) include headache, dizziness, feeling sick, vomiting, abdominal pain, loss of appetite, palpitations, pain in muscles and joints, fatigue and disturbed sleep. Commonly (greater than 1 in every 
10 patients treated) reported side effects include alterations to the electrocardiogram (ECG), tingling in hands and feet, problems with walking, cough, diarrhea, itching, rash and insomnia. Uncommon side effects (greater than 1 in every 1000 patients treated but less than 1 in 100): involuntary muscle jerks, coordination disturbances, altered liver function tests and drowsiness. The following side effects have been reported in patients treated with Lumartem. However, frequency estimates for these side effects are not available: hypersensitivity reaction, hives, rapid swelling of the face and throat (angioedema). A similar side effect profile was reported for children.

RESULTS:

Flesch Reading Ease score: 35.9 (text scale)

Flesch Reading Ease scored your text: difficult to read.

Gunning Fog: 15.3 (text scale)

Gunning Fog scored your text: hard to read.

\section{BRAND NAME: SANOFI AVENTIS}

Before you take Artesunate Amodiaquine Winthrop. Do not take Artesunate Amodiaquine Winthrop if you are allergic to artesunate amodiaquine or any of the other ingriedients of Artesunate Amodiaquine Winthrop (see section 6, What Artesunate Amodiaquine Winthrop contain). - If you have ever had liver problems during treatment with amodiaquine. - If you have ever suffered from sudden high fevers, shaking, severe sore throat or ulcers in the mouth (symptoms suggestive of harm to white blood cells) during treatment with amodiaquine. -If you have an eye disease with damage to your retina. - If you have any doubt, it is essential to ask the advice of your doctor pharmacist or health care worker. Take special care with Artesunate Amodiaquine Winthrop. This medicine may cause damage to the liver and/or blood that can sometimes be fatal in case of marked asthenia (fatigue), gastrointestinal disturbances, (nausea, vomiting, abdominal pain), jaundice (dark urine, pale bowel motions) sore throat or mouth ulcers, stop the treatment and consult a doctor immediately; you doctor may need to take a blood sample. Tell your doctor, pharmacist or health care worker, if you have used this or another amodiaquine-containing medicine very recently. Since you might then be more likely to experience side effects, special caution is warranted. Because caution should be exercised in some patients, it is important to inform your doctor if you have or have ever had, any of the following: a problem with your heart rhythm or any other heart problem.

\section{RESULTS:}

Flesch Reading Ease score: 38 (text scale)

Flesch Reading Ease scored your text: difficult to read.

Gunning Fog: 14.5 (text scale)

Gunning Fog scored your text: hard to read. 